\title{
Cellular Automata - a Tool for Disorder, Noise and Dissipation Investigations
}

\author{
W. Leoński ${ }^{1}$ and A. Kowalewska-Kudłaszyk ${ }^{2}$ \\ ${ }^{1}$ Quantum Optics and Engineering Division, Institute of Physics, \\ University of Zielona Góra, Zielona Góra \\ ${ }^{2}$ Department of Physics, Adam Mickiewicz University, Poznań
}

Poland

\section{Introduction}

There are many problems that are impossible or very difficult to solve with numerical methods. In particular, they are related to the systems containing many subsystems for instance, atoms, molecules or even water drops and sand grains. Moreover, to this group we can number many continuous systems that are treated as a discrete set of many sub-systems. Although the dynamics of many of them can be described by the set of differential equations, such attempts are futile. Due to the complexity of the whole system, finding solutions for such huge amount of equations is often impracticable. For these cases the cellular automata (CA) formalism seems to be one of possible solutions.

Although the idea of CA was proposed during II World War in the Los Alamos laboratory by Stanisław Ulam and Janos von Neumann, for its real development and practical applications we had to wait three decades (for references concerning those early papers concerning CA simulations see for instance Butler (1973) and the references quoted therein). In consequence, from the 1970-s we can record growing number of papers devoted to CA applications in various fields of physics, biology, medicine, sociology, economy and other. For instance, one should mention the paper by Margolus et al. (1986) who applied CA for the fluid dynamics modelling. CA formalism can also be used for Brownian motion investigation, for instance for the solid-fluid suspensions (Ladd \& Colvin (1988)). For solid state systems CA formalism allows simulation of magnetisation processes (Vichniac (1984)). Moreover, CA investigation concerning laser dynamics was presented by Guisado et al. (2003), whereas N-body systems with multi-step potentials were discussed by Lejeune et al. (1999). It should be emphasized that CA formalism was applied not only for physical systems investigation but also for medical (for instance see Barclay et al. (2010)), sociological (Dabbaghian et al. (2010)), traffic flow (Combinido \& Lim (2010)), agriculture (Zeng et al. (2010)), image processing (Rosin (2010)) and others. These are only some selected recent examples of the papers dealing with a very broad and diverse subject of CA applications.

CA can be defined as dynamical system that is discrete in time and space and is characterized by discrete states. This rather general description is far from the mathematical exactness and precision however, it reflects the basic properties of CA. Of course one should realize that there are various strict mathematical CA definitions. At this point we shall present such CA 
definition that is not mathematically pure, but can be readily applied by the reader in his further investigation. So, the cellular automaton $\mathcal{A}$ can be defined as:

- $n$-dimensional lattice of cells $\mathcal{L}^{n}$,

- the finite set $\mathcal{S}$ of states of the cell,

- the neighbourhood of the cell $\mathcal{N} \subset \mathcal{L}^{n}$,

- the rules determining the state of given cell in the next time-step on the basis of its current state and the state of its neighbours.

In this paper we shall concentrate on practical realization of a such defined CA. We intend to show how such simple CA definitions allow construction of the model reflecting physical properties of the real system. Moreover, our aim was to present how a complicated system evolution can be investigated with the help of CA. In particular we shall discuss the model of many two-level subsystems. Two-level systems were used and discussed extensively in physical models of solid state physics or quantum optics (for discussion concerning the latter see the classical book by Allen \& Eberly (2007)). Obviously such two-level systems can also be discussed as sociological or economical models. For instance, two-level 0-1 system may be related to the voting or sell-buy decisions. At this point it is worth mentioning the Sznajd model (Kondrat \& Sznajd-Weron (2010) and the references quoted therein) describing physical Ising model and the sociological voting one as well. In general, one can say that many two-level system models can be applied to the energy or information dynamics simulations.

\section{The model}

In this chapter we shall concentrate on one-dimensional simple model comprising many two-level subsystems. This model was initially proposed by Walczak \& Leoński (2003), where the first, preliminary considerations were presented. The Authors concentrated on the relations between the model proposed and a cavity with two-level atoms dynamics. They discussed the dependence of the system energy on the absorption probability for the individual atom. Moreover, for this model the entropic measures of disorder was defined and discussed in the papers Kowalewska-Kudłaszyk \& Leoński (2006; 2008).

The model is organized as follows. First, we assume that we have a chain of two-level subsystems (atoms, cells). Each of them can be in one of the two states: ground or excited. These states will be denoted by 0 and 1, respectively. Obviously, depending on the application of our model, the states can be labelled for instance, as "up" and "down", "buy" and "sell" etc. Each of excited atoms can "emit energy (photon)" toward one of the two directions (left or right). If such emitted quanta of energy meet the atom in the ground state, it can absorb the energy. Since, our model has the probabilistic character, we assume some values for probabilities of the energy absorption and emission, and for the direction of the emission as well.

At the beginning of our numerical experiment we assume some length $N$ of the system considered (number of cells) and the values of the emission and absorption probabilities: $p_{e}$ and $p_{a}$, respectively. These probabilities are identical for all cells and remain constant during the whole process. Moreover, we assume which cells are initially excited. This initial cells states selection can be either done by some assumption (deterministic initial conditions) or performed randomly (random initial conditions).

Then we determine the simulation rules and thus, our simulation algorithm can be summarized in the following points: 
- If for the time $t$ particular atom $\{i\}$ is in its excited state, we take some random number $r_{i t} \in<0,1>$. If $r_{i t}<p_{e}$ the atom in the next step (time $t+1$ ) emits quantum of energy.

- If the energy is emitted, we take another random number $r_{j} \in<0,1>-$ for $r_{j}<1 / 2$ the quantum goes left (for $r_{j} \geq 1 / 2$ it goes right).

- If the photon meets the excited atom it passes this cell and goes farther (the atom is "transparent for radiation").

- If the quantum reaches the atom in its ground state we take the next random number $r_{j t} \in<0,1>$. If $r_{j t}<p_{a}$ the atom in the next step (time $t+1$ ) absorbs photon. For the opposite case the photon goes to the next cells.

The next point is determination of boundary conditions of the model discussed. Thus, we shall consider two cases. For the first of them (periodic boundary conditions) we assume that the first and the $N^{\text {th }}$ cells are the same. It corresponds to the real systems of the ring cavities commonly discussed in optical models. For this case the total "energy" of the system is preserved, since we neglect all dissipation processes. Such "energy" can be calculated as a sum of all excited atoms at the given moment of time located within the system considered. The other possibility is to assume that the energy can leak out of the system. For this case we can assume that at the ends of the cavity we have semi-transparent "mirrors". According to it we need to choose some reflection probability $R$ at the beginning of the simulation. We assume that if the quantum of energy ("photon") reaches the "mirror" confining the cavity, we take a random number $r_{R} \in<0,1>$. For the case when $r_{R}<R$ the photon remains inside the cavity and start to move in the opposite direction. If $r_{R} \geq R$ the quantum of energy disappears and the total system energy decreases.

One can see that our model has discrete character. Both time $t$ and the position of the cell are discrete, which is characteristic of CA. Thanks to this fact, the parameters describing the system will change jumping from one value to another showing step-function character. Since we need continuously changing parameters we should avoid such behaviour. Therefore, we shall perform many simulations for the same values of the initial parameters and then, average the results. This problem was discussed for the case of the system energy in the earlier mentioned (Walczak \& Leoński (2003)), where the first description of the model considered was presented.

\section{Simulation results}

At the beginning we present the exemplary simulation results for the system confined by "mirrors" with the reflection probability $R$ assumed to be equal 0.25 . We deal with a simple 13-cells model assuming that only three of them are initially excited. From Fig.1 one can see that for the time $t=0$ we have excitations at the cell positions 9, 10 and 11. During the evolution, these excitations walk randomly right and left reaching the borders of the automaton in the time equal to 46 (right) and 51 (left). Since the reflection probability has a non-zero value, the "photons" are reflected and the system's energy is conserved. However, for $t=58$ (right) and $t=59$ (left) both energy quanta are emitted from the system. As a result from $t=60$ we observe random walk of only one excitation.

\subsection{Rate of energy leakage}

At this point we shall concentrate on the CA model corresponding to the cavity with "mirrors" and we assume that the reflection probability $R$ is smaller than unity. Since such a model can include dissipation effects, it allows the total energy leakage investigation. In particular, we 


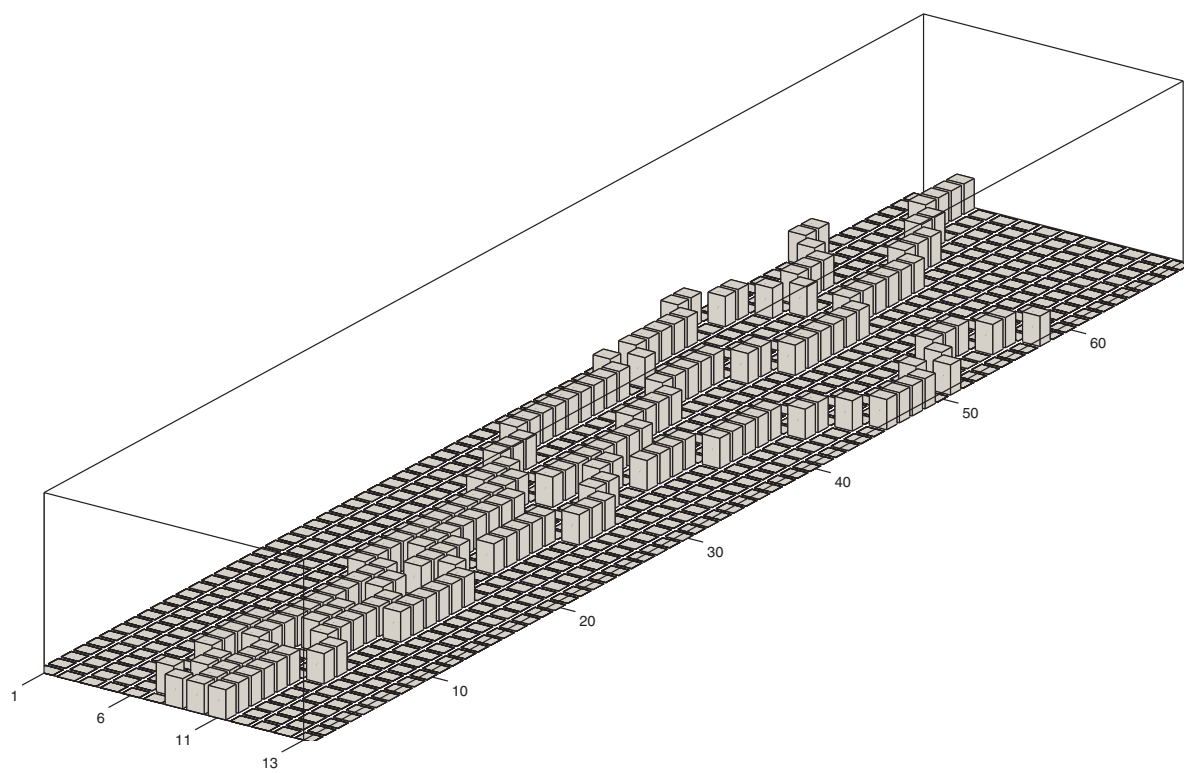

Fig. 1. A map visualising the spread of excitation from the three initially excited cells with time (from 1 to 70 ) for $p_{a}=1.00, p_{e}=0.25$ and the reflection probability $R=0.25$. CA contains 13 cells.

shall be interested in the energy loss rate. Analysis of this rate behaviour can be a good test of whether our model reflects the properties of the real systems properly or not.

Therefore, at this point we define the rate related parameter $r=E_{n+1}-E_{n}$ as a differences between two subsequent total system energies. We do not divide such difference by the time, as it is equal to unity. This is a result of the discrete character of CA - within CA models time is discrete and numbered by the subsequent natural numbers.

It should be stressed out that to find the time-dependence of $r$ we have to perform a series of simulation experiments for the same values of parameters describing the system. For a single simulation we obtain the energy time-dependence of the step-like function character. Therefore, to obtain a sufficiently smooth form of the results, we performed some number of simulations (for our case it was $\sim 100$ ) and then, we averaged the results. Sometimes such averaging procedure was not sufficient and we needed additional smoothing procedure application. For the cases discussed here we applied LOESS (or LOWESS) - locally weighted scatterplot smoothing procedure (Cleveland (1979); Cleveland et al.\& Devlin (1988)). This method allowed correct calculations of $r$ without losing features appearing in the results. At this point we shall concentrate on the deterministically chosen initial conditions. Thus, we assume that our model contains 150 cells. Those labelled by numbers 51-100 (located at the centre of the line) are excited, whereas the remaining ones are in their ground states. We assume that the absorption probability $p_{a}=1.00$. Moreover, the probability of emission is assumed to be equal to 0.25 . 


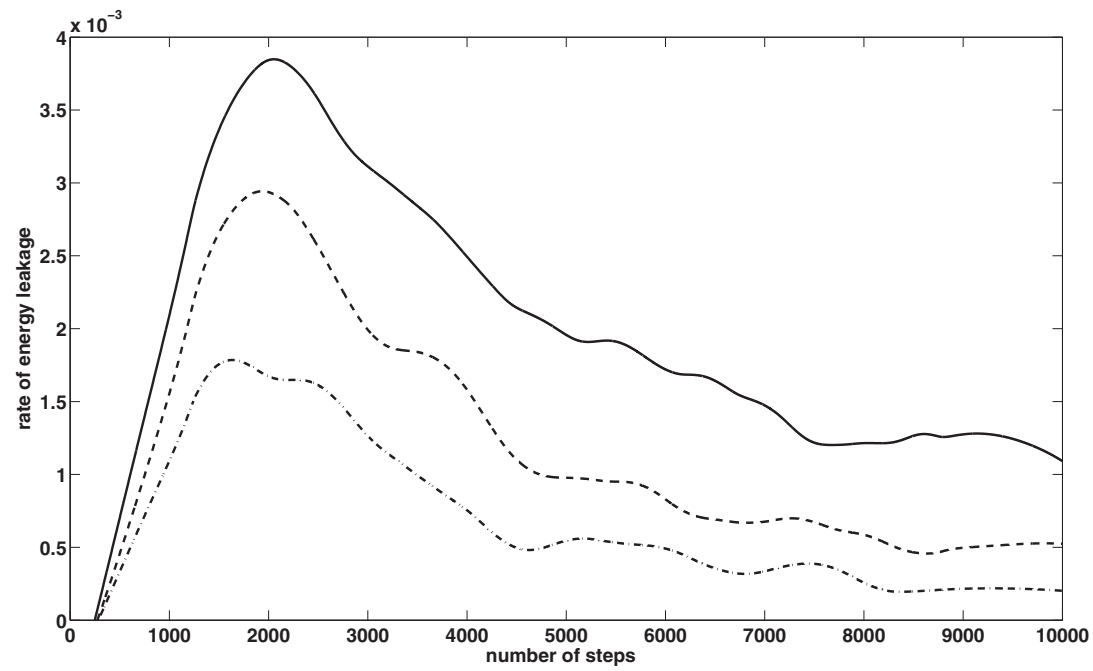

Fig. 2. Rate of energy leakage from the cavity for various values of the mirror reflection probabilities: solid line $-R=0.25$, dashed line $-R=0.50$ and dashed-dotted line $-R=0.75$. We assume the deterministic initial conditions and the probabilities $p_{a}=1.00, p_{e}=0.25$.

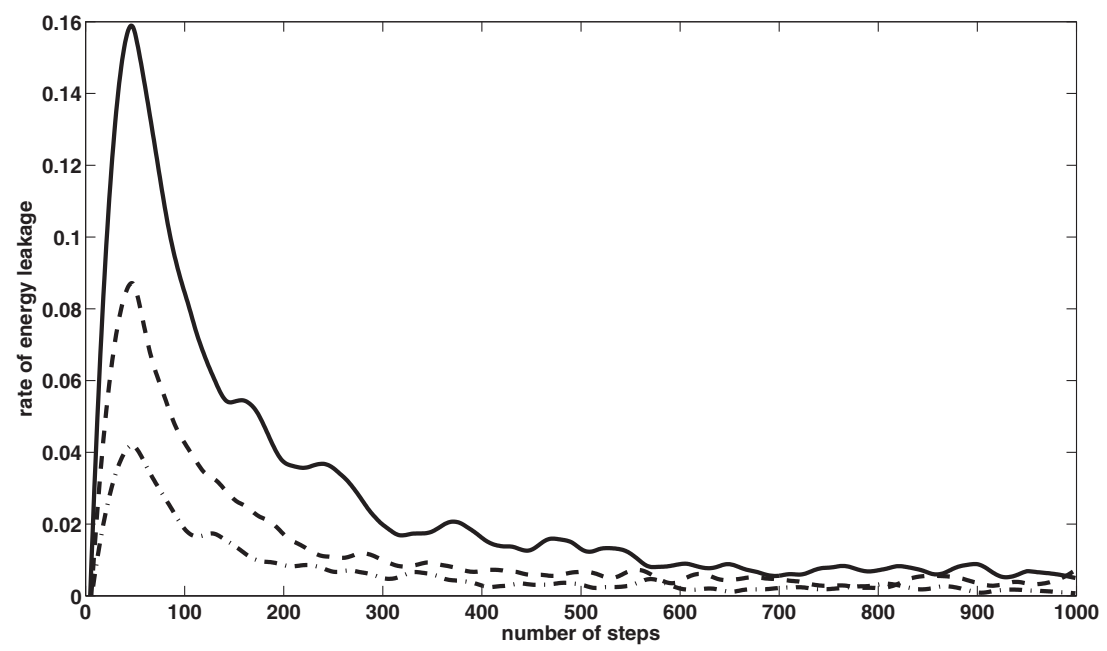

Fig. 3. The same as in Fig.2 but for $p_{a}=0.25, p_{e}=1.00$. 
Thus, Fig. 2 shows the energy leakage rates for various values of the reflection probability for the mirrors confining the system. We see that the rate $r$ is equal to zero for approx. 300 first steps. This is related to the fact that the excited cells are located in the central part of the automaton. Therefore, the excitations need some period of time to reach the "mirrors". After that the value of $r$ increases rapidly reaching some maximum and begins to fall. This rate decay resembles an asymptotic decay. It means that the system relatively fast loses its energy and after that if there are very few excited cells left, the energy of the whole system does not change a lot. From Fig.2 one can see that the lower the reflection probability $R$ the higher values of the rate $r$ are achieved. Moreover, we see that the length of the initial period of time when $r=0$ does not depend on the value of $R$. These features are in a good agreement with the expected behaviour of energy in real cavity systems. This fact strengthens our conviction about the correctness of our model.

Fig. 3 shows the situation analogous to that shown in Fig. 2 but for $p_{a}=0.25$ and $p_{e}=1.00$. This means that every cell that is excited for a given time-step emits energy quantum immediately (in the next time-step). Generally, in Fig.3 we see the same features as those shown in Fig. 2 but for this case all processes are more rapid in character. As we compare the time-scales of these two plots we see that for the case discussed here we deal with processes one order of magnitude faster then previously. Moreover, the values of the energy leakage rate are two orders of magnitude greater than for the cases shown in Fig.2. This is not only an effect of the immediate energy emission by excited cells. As shown in paper (Kowalewska-Kudłaszyk \& Leoński (2008)), absorption of energy by the cells that are in their ground states can play a dominant role in the system. This effect was referred there as to molasses effect.

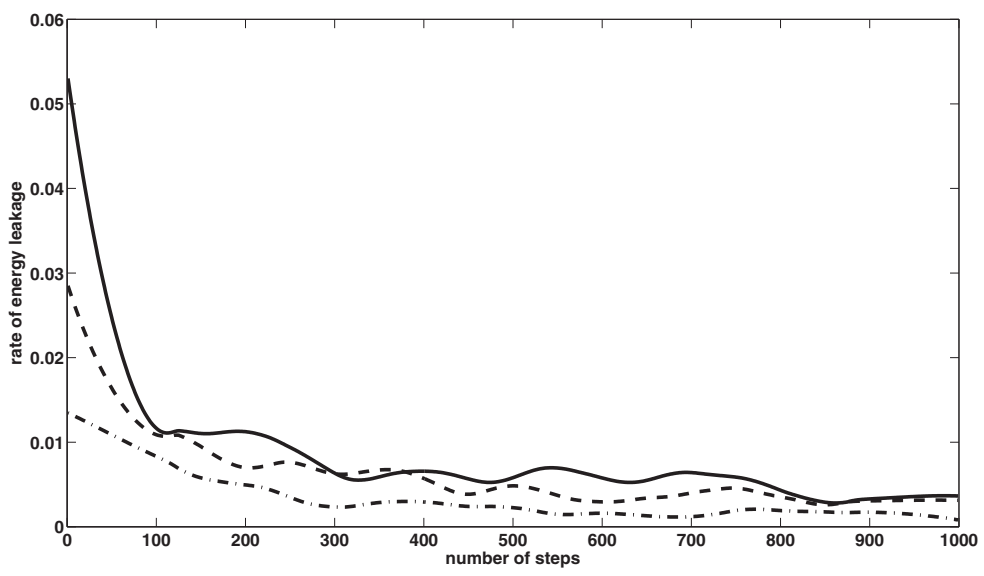

Fig. 4. The same as in Fig.2 but for random initial conditions.

Finally, we show the energy leakage rate $r$ time-dependence for random initial condition. We assume that for the time $t=0$ we have 50 excited cells again but they are randomly spread across the whole automaton line. All parameters are assumed to be equal to those from Figs.2 and 3. Thus, Fig.4 corresponds to the situation depicted in Fig.2, whereas Fig.5 is related to Fig.3. In both figures (Figs.4 and 5) one can generally see the behaviour of $r$ analogous to that discussed previously (Figs.2 and 3, respectively). However, for the case of random initial 
condition, $r$ starts its evolution from non-zero maximal values decreasing with time. We do not observe the initial "dead" period when $r=0$. Such a behaviour is related to the fact that all excitations are already spread across the automaton line for $t=0$. As a result, the excitations do not need any initial period of time to reach the cavity border ("mirror").

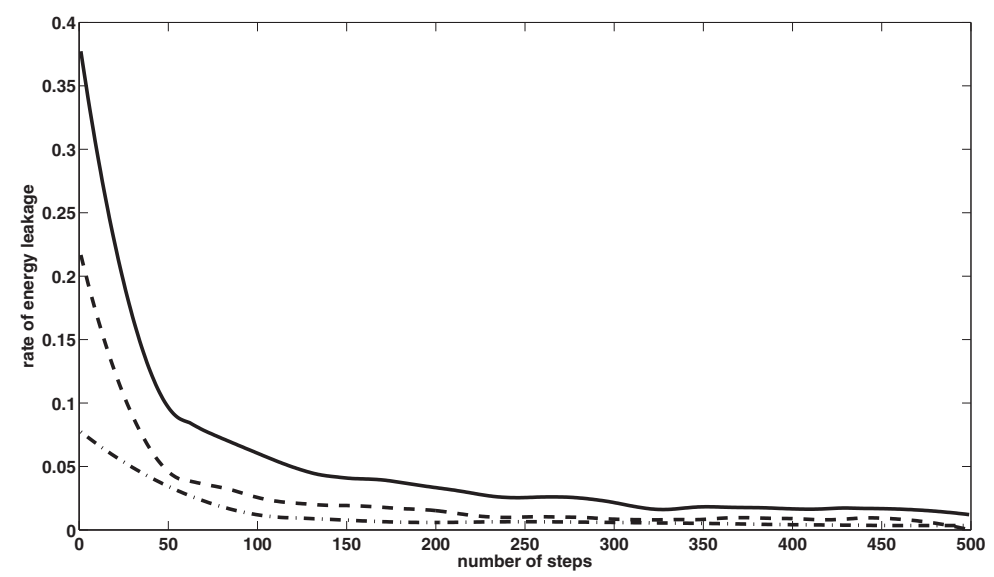

Fig. 5. The same as in Fig.3 but for random initial conditions.

\subsection{Entropic disorder measure and autocorrelation function}

As we assume that the reflection probability $R$ is equal to 1.00 or(and) we deal with periodic boundary conditions we remove all dissipation effects from our model and the total energy of the system remains constant (the rate $r=0$ ). For such a case the model becomes a very good tool for investigation of disorder. If the excitations are initially grouped in a given part of the automaton after some time of evolution they are randomly spread over the whole system. As a result we start from an ordered system and end at disordered one. Obviously, it is possible to include dissipation to the model and we shall do it for the cases discussed here. However, at this point it should be mentioned that if the total number of excitations decreases, some problems with determination of whether the system is ordered or not can occur (this fact was discussed in the paper Kowalewska-Kudłaszyk \& Leoński (2008)).

Although it is possible to measure the degree of ordering of our system with application of various parameters, we shall concentrate on that proposed in our earlier papers (Kowalewska-Kudłaszyk \& Leoński (2006; 2008)). We have proposed and discussed the entropic parameter $\mathcal{E}$ defined on the basis of the Fourier transform

$$
F(\omega)=\int_{x_{0}}^{x_{\text {end }}} f(x) \exp (-i \omega x) d x,
$$

where the function $f(x)$ is equal to zero for the cells in their ground states or to unity for the excited cells. We perform integration over the whole length of the automaton and $x$ labels the cell position. Due to the discrete character of CA we replace this integral by the discrete sum as follows

$$
F(\omega)=\sum_{x=x_{0}}^{x_{\text {end }}} f(x) \exp (-i \omega x)
$$


Applying thus defined function we introduce the "power spectrum" $P(\omega)=|F(\omega)|^{2}$. Next, we normalize it and define the "entropy" of the system discussed as

$$
\mathcal{E}=\sum_{\omega} P_{N}(\omega) \log (P(\omega))
$$

where we perform the summation over the all frequencies appearing in the system. The index " $N$ " appearing here means that the spectrum is already normalized. To avoid interpretation problems related to the influence of the total number of excited cells on the value of entropy we shall deal with the "entropy per number of excited cells". Practically, for each time-step we divide the parameter $\mathcal{E}$ by the current number of excitations in the system.

Thus, Fig. 6 shows the time-evolution of $\mathcal{E}$ for various values of the reflection probability $R$. Moreover, we assume that the absorption probability is small $\left(p_{a}=0.25\right)$ and the excited cells emit energy immediately $\left(p_{e}=1.00\right)$. For the time $t=0$ all excitations are assumed to be located centrally, i.e. we have 50 excited cells at the locations 51-100 (cells 1-50 and 101-150 are in their ground states). From Fig. 6 we see that for $R=0.50$ and 0.75 the time-evolution of $R$ can be divided into two stages. First, we see its rapid growth and then (after approx.100 time-steps) almost linear - very slow increase in the value of $R$. This slow increase can be practically neglected and the entropy can be treated as constant (for the discussed period of time). However, if we assume that $R=0.25$ the third, intermediate stage is apparent. For the time-steps $\sim 20-300$ we observe the smooth, nonlinear increase in the entropy. After that we can see the linear increase again, however, the increase-rate is considerably greater than for the cases of greater values of $R$.

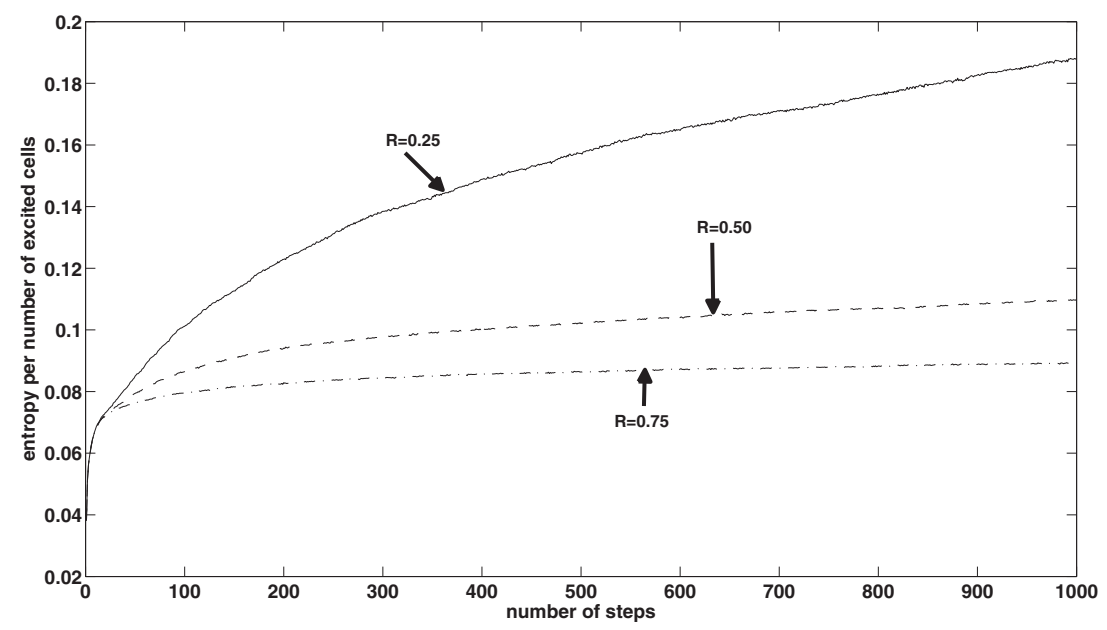

Fig. 6. The entropies for various values of the reflection probability $R$. Other parameters are: $p_{a}=0.25, p_{e}=1.00$. Random initial conditions are assumed.

Fig.7 shows the opposite, situation - we assume that the absorption probability is equal to unity whereas, $p_{e}=0.25$. We see that the first step (rapid, initial growth of the entropy) and the second one (nonlinear growth) are identical for all values of $R$. However, for the time-steps above $\sim 1500$ we see a linear increase in entropy but at different rates for different 


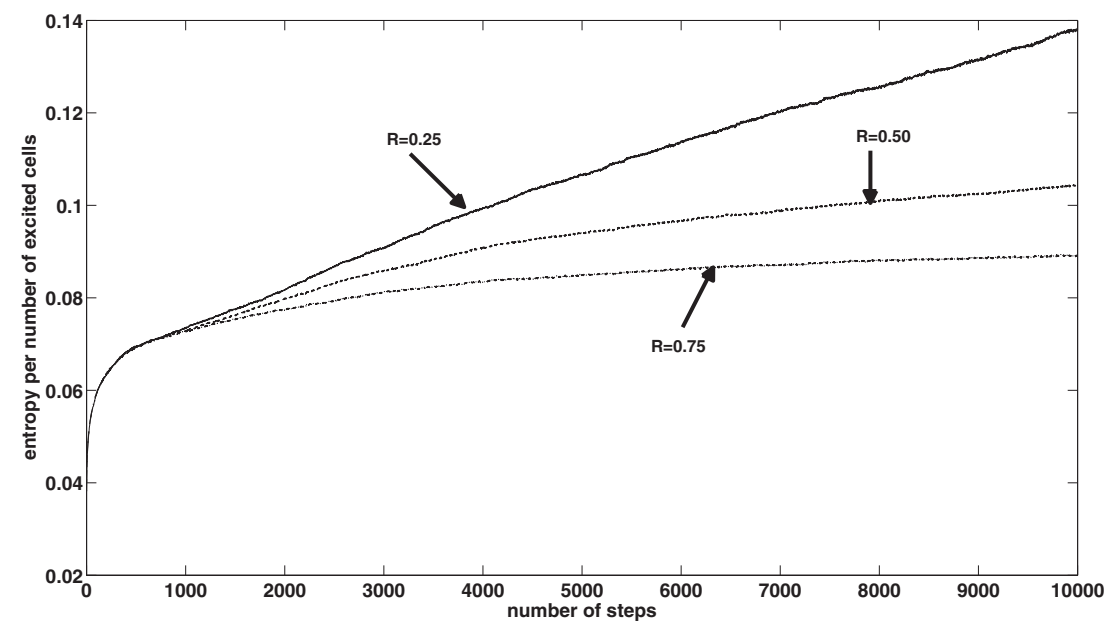

Fig. 7. The same as in Fig. 6 but for $p_{a}=1.00$ and $p_{e}=0.25$.

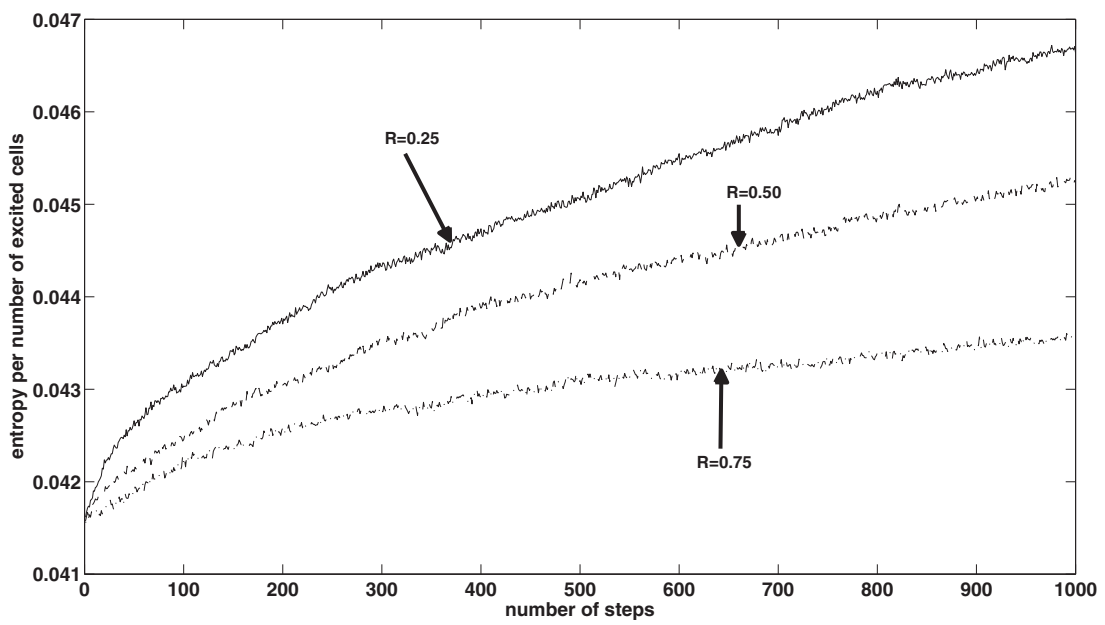

Fig. 8. The same as in Fig.7 but for random initial conditions.

values of the reflection probability. For $R=0.50$ and 0.75 we observe slowly growing value of $\mathcal{E}$ whereas for $R=0.25$ the increase is more pronounced. This behaviour is similar to that shown in Fig. 6, but one should note that the time-scale we discuss here is by one order of magnitude greater then previously. This is a result of the fact that the absorption probability is assumed to be unity. For this case the whole system's evolution slows down considerably and this is the mentioned earlier molasses effect. 
Fig.8 presents the time-evolution of $\mathcal{E}$ for random initial conditions. For this case it is assumed that for $t=0$, the excitations are randomly distributed along the whole system. As a result, the initial values of entropy are comparable to its final ones, contrary to the previous cases (deterministic initial conditions) where $\mathcal{E}$ started its evolution practically from zero value. Moreover, the entropy time-variations have noisy character and now we shall concentrate on it.

One of the methods for investigation of systems in which some kind of disorder appears is that based on the autocorrelation function (AF). This function, frequently used in signal processing, measures the existence of correlations of the signal with itself but shifted in time scale backwards for a specified time value. The function can confirm the existence of any repetitive patterns in the signal analysed, even if they are obscured by any kind of noise that affects the signal considered.

AF can be used to trace the degree of disorder that emerges during evolution of the system we are dealing with. If our system is initially ordered, we can try to find whether during the evolution under various conditions applied (as absorption and emission probabilities or refraction probability of resonator mirrors), the system becomes disordered or not. If there are such conditions that would force the excitations to spread inside the resonator in any periodic way, the autocorrelation function notes that fact.

The definition of the autocorrelation function used comes from the relation (Bendat \& Persol (2010)):

$$
C(\tau)=\frac{1}{N-1-\tau} \sum_{i=0}^{N-1-\tau} a_{i} \cdot a_{i+\tau},
$$

where $\tau$ is the time delay, $N$ is the number of elements in series and $a_{i}$ is the value of the $i-t h$ element of the series analysed. If the autocorrelation coefficient is to be normalised, it should be additionally divided by the value of $C$ for $\tau=0$ and in this paper such normalised AF is used.

For the cases discussed here the autocorrelation function will be applied as a measure of correlations between the entropies corresponding to various moments of time. Autocorrelation function measures the character of changes in entropy with time. We shall examine two extreme cases for both random and deterministic initial conditions: the former corresponds to maximal absorption and small emission probabilities (Fig.9 and Fig.11) and the latter to small absorption and maximal emission probabilities (Fig.10 and Fig.12).

We start our consideration from the case when the molasses effect is dominant in the system. This situation corresponds to the assumed maximal absorption and relatively small emission probabilities. For deterministic initial conditions (Fig.9) we can see slow decay of AF with increasing time-shift $\tau$ parameter, since the entropy is a slowly increasing function of time. It means that the system during its evolution becomes more disordered than it was at the beginning, but this process is rather slow. In contradiction to the opposite probability values (Fig.10 $-p_{a}=0.25$ and $p_{e}=1.00$ ) we can observe practically no changes in longer time. The reason is that the entropy reaches its high value almost instantly and the system initially ordered very quickly becomes disordered. After that any changes in $\mathcal{E}$ are rather slow in character as we compare them with its initial growth. For this case we can differentiate AF corresponding to various values of $R$ much more easily than for the previous case. This is a result of the fact that for this case AF is almost constant and when the value of $\tau$ becomes 


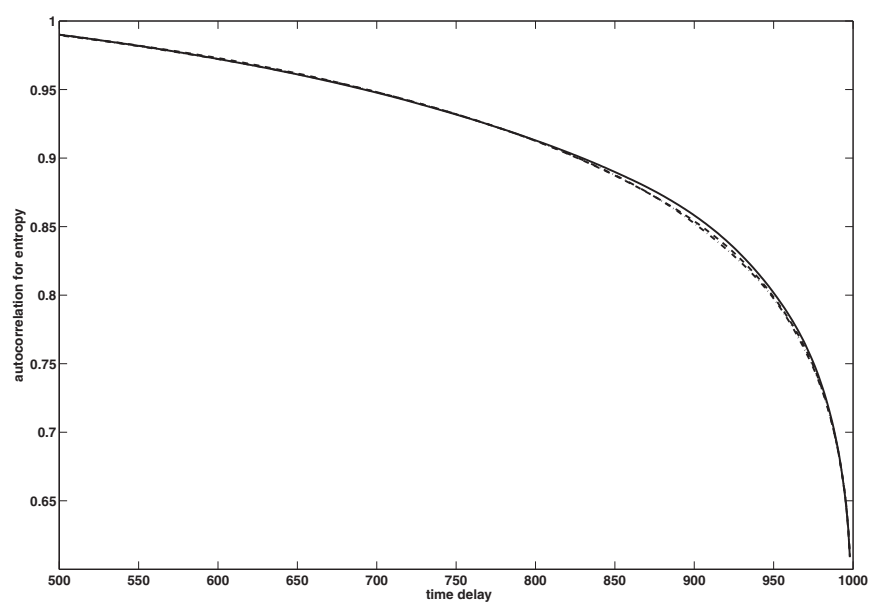

Fig. 9. Entropy autocorrelation function v. time-delay parameter $\tau$, for various values of the reflection probability $R$ : solid line $-R=0.25$, dashed line $-R=0.50$ and dashed-dotted line $-R=0.75$. Other parameters are: $p_{a}=1.00, p_{e}=0.25$. Deterministic initial conditions are assumed.

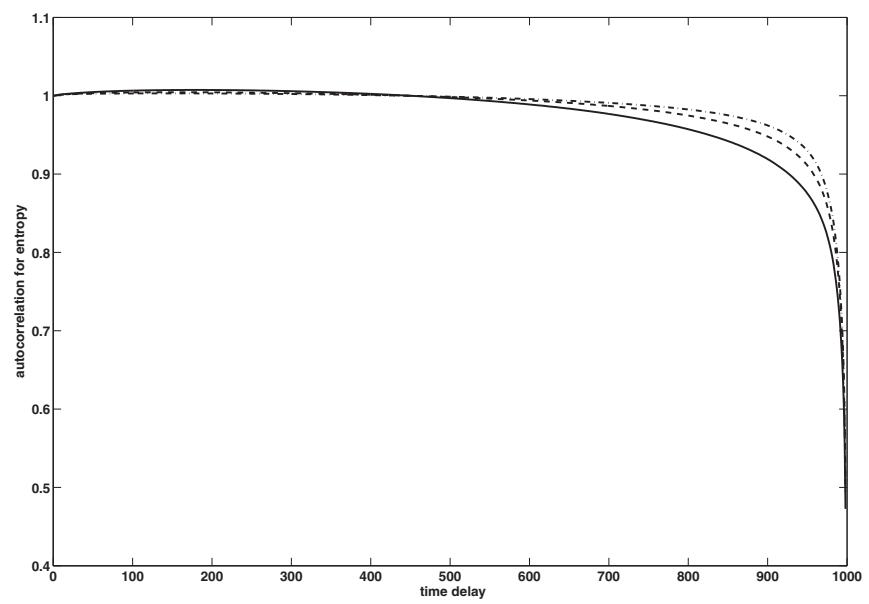

Fig. 10. The same as in Fig. 9 but for $p_{a}=0.25, p_{e}=1.00$ and various values of $R$ : solid line $R=0.25$, dashed line $-R=0.50$, dashed-dotted line $-R=0.75$. 


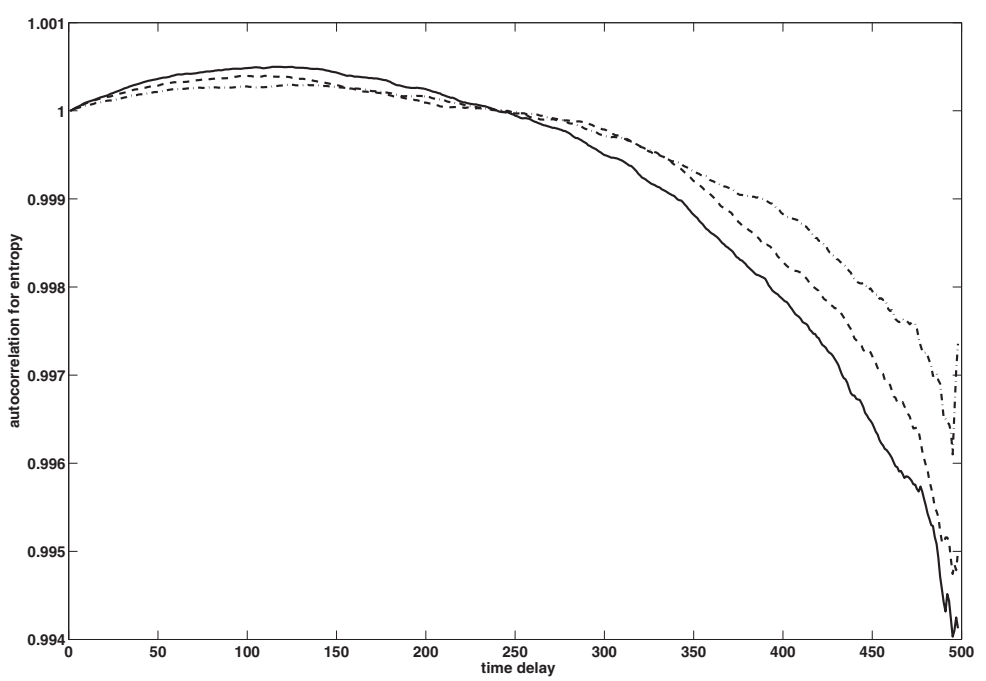

Fig. 11. The same as in Fig.9 but for random initial conditions.

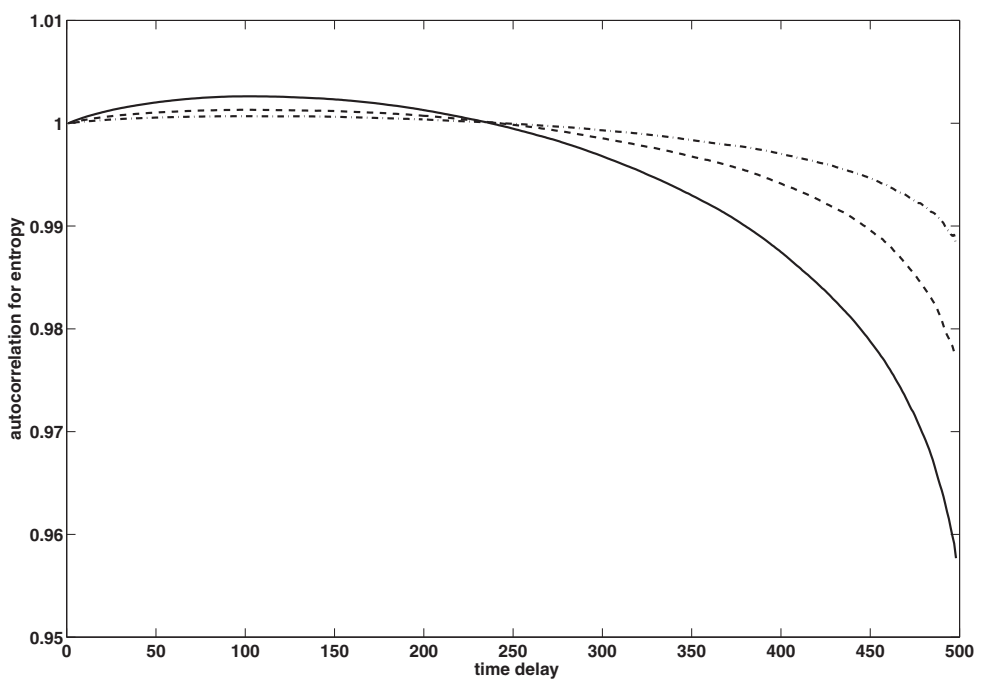

Fig. 12. Entropy autocorrelation function for the same parameters as those of Fig.10 but with random initial conditions. 
close to the cavity dimension this function falls rapidly. This decrease appears more suddenly and is more rapid as the probability of reflection increases. Such behaviour is caused by greater velocity of the excitation movement - the excitations reach the "mirrors" faster than it was for maximal absorption probability case. This leads to the visible differences in the dynamics of energy leakage from the cavity.

The analogous features can be seen for the random initial conditions cases (Fig.11 and 12), but the changes in AF are very tiny as we compare them with those corresponding to the ordered, deterministic initial conditions. One should remember that the excited cells are initially spread along the whole cavity. Even when the absorption probability reaches its maximal value, the energy quanta can escape from the cavity sooner than it was for the case of deterministic initial conditions. For both values of absorption and emission probabilities we can also see some initial small growth in AF but one should keep in mind that the vertical axes scale appearing in Figs.11 and 12 is much more stretched as we compare it with those in Fig.9 and 10. However, such small increase in the AF value is an effect of short-time entropy vs. time regular dependence at the beginning of the entropy growth process. However, after this short period of time, entropy becomes an irregular function of time and AF decreases.

\subsection{Recurrence plots}

As shown, the system discussed here can exhibit complicated evolution. To determine its character, we can apply the recurrence plots (RP) analysis. This investigation method allows determination whether the system evolves chaotically, exhibits regular or periodic dynamics or finally, is subjected by some noise. Moreover, RP can be applied for the situations when we cannot obtain sufficiently long time-series. For explanation of the RP idea one can see the review paper Marwan et al. (2007) and the references quoted therein. The idea of RP was proposed by Eckmann et al. (1987) and is widely used in nonlinear data analysis up to now. To build RP we need to find the binary matrix. Its elements are defined by the formula (Eckmann et al. (1987)):

$$
R_{i, j}=\Theta\left(\epsilon_{t h r}-\left\|\vec{x}_{i}-\vec{x}_{j}\right\|\right), \quad i, j=1, \ldots, N, \quad \vec{x}_{i}, \vec{x}_{j} \in \mathcal{R},
$$

where $\Theta$ is the Heaviside function, $\epsilon_{t h r}$ is the threshold parameter, and \|\| denotes the norm. This norm allows determination of the distance between two points. To determine this matrix we need to reconstruct the trajectory in the phase-space on the basis of the time-series we investigate. The modulus $\left\|\vec{x}_{i}-\vec{x}_{j}\right\|$ determines the distance between the points in the reconstructed phase-space. If two points fall inside the same region (sphere of the radius $\left.\epsilon_{t h r}\right)$ they are labelled by 1 , otherwise they labelled by 0 . Applying this procedure we obtain a matrix filled with zeros and ones that can be illustrated by white and black point. If we reconstruct the phase space in which the system analysed lives, we look for times at which system returns to the same area of that phase space. In fact, RP shows whether the system analysed via the time series inspection recurs or not. Analysis of the so obtained matrix (picture consisting of the black and white points) allows determination the dynamics we are dealing with.

In this chapter we present RP for automaton determined by random initial conditions where the excited cells are randomly spread over the whole system. Moreover, we shall discuss the cases of the cavity with cyclic boundary conditions and that of the cavity confined by the "mirrors". Since, the energy of such a cyclic model is preserved we shall concentrate at this point on the entropic parameter $\mathcal{E}$ time-evolution. This "entropy" describes how disordered our system is and RP based entropy analysis gives us information about the character of 
changes in $\mathcal{E}$. The type of such variations should reflect the character of the whole system's dynamics. Excitation movements along the "cavity" are determined by the absorption and emission probabilities that influence the derived RP as well. RP derived on the basis of our model should clarify the nature of the system evolution despite the complex form of this evolution. Many features are obscured by the random processes taking place in the system and we shall show that RP can reveal them.

At first, let's concentrate on the cyclic boundary conditions and compare two extreme cases: maximal absorption with small emission and maximal emission with small absorption probabilities. The time-evolutions of entropy and the corresponding RP for all cases discussed in this section are plotted. The character of evolution of $\mathcal{E}$ is similar to those discussed in the previous section (Figs.15 and 16) if we assume that the system is confined by the mirrors. For cyclic boundary conditions, the entropy exhibits random deviations from some constant value. If we neglect them the entropy can be treated as constant (note the scale of vertical axis in Figs.13 and 14). However, the question arises whether these random deviations originate from noise effects or some deterministic chaotic features can be expected. Moreover, it would be desirable to check whether any quasi-periodic or periodic evolution are hidden behind the "noisy" view of $\mathcal{E}$ evolution. Therefore, these entropies are plotted with the corresponding RPs.

As follows from the recurrence plot shown in Fig.13 $\left(p_{a}=1.00, p_{e}=0.05\right)$, the entropy evolution has a rather noisy than chaotic character. Although some diagonal lines appear, but they are formed of a few points only (average: 2.5 points). Since the length of the diagonal lines is related to the value of the sum of positive Lyapunov exponents, short lines indicate that we have not chaotic behaviour in this case. We see that in the system the noise effects are dominant over the chaotic ones. This observation agrees with the fact that for the situation discussed here, the fraction of all of the points that form the diagonal lines is about $8 \%$. This indicates that a significant number of the points that recur are practically isolated ones. All these features confirm that the entropy changes are of noisy character. For the case when $p_{a}=0.05, p_{e}=1.00$ (see Fig.14) again the entropy vs. time-evolution of $\mathcal{E}$ should be classified as a noisy signal. For this case the percent of points forming diagonal lines is smaller than it was observed previously. It means that for both extreme cases initial random conditions result in a noisy character of entropy dynamics.

Next, we change the boundary conditions from the cyclic ones to those corresponding to the cavity with mirrors. Although for this case the dissipation processes is included, we shall discuss the character of the entropy evolution again. This allows a comparison of the results with those discussed above for the cyclic conditions. Fig. 15 shows the entropy evolution and the corresponding RP for the probability of reflection $R=0.75$. Moreover, it is assumed that $p_{a}=1.00, p_{e}=0.10$ and the cells are initially randomly excited. The plot of $\mathcal{E}(t)$ (Fig.15 - top) has the same irregular character as that for the cyclic conditions. The only difference is in the initial growth of entropy. Nevertheless, from the form of this plot we are not able to say anything on the character of the time-evolution. However, analysis of RP (Fig.15 - bottom) shows that the dynamics of the system differs considerably from that discussed earlier. Both horizontal and diagonal lines forming rectangular structures are observed. They are dominant over single dots characteristic of noise, and they are a result of some periodic and quasi-periodic effects and drift ones (logistic map is corrupted with a linearly increasing term). Moreover, the plot shows some features characteristic of the Brownian motion. Periodic effects are related to the finite length of the cavity and a finite constant velocity of the excitation 

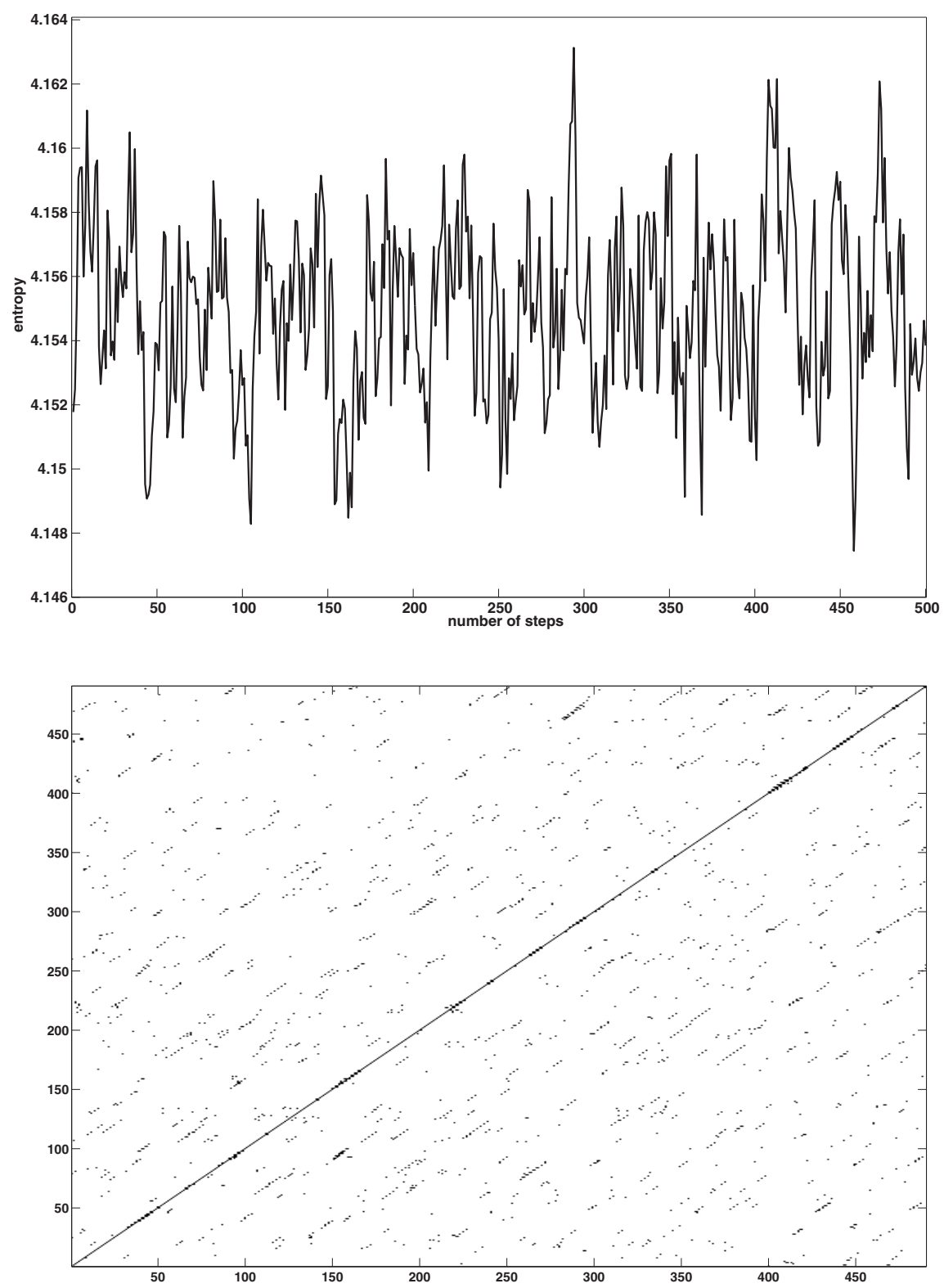

Fig. 13. At the top the entropy for $p_{a}=1.00$ and $p_{e}=0.05$ and cyclic boundary conditions. At the bottom the recurrence plot corresponding to the entropy evolution. We assume random initial conditions. 

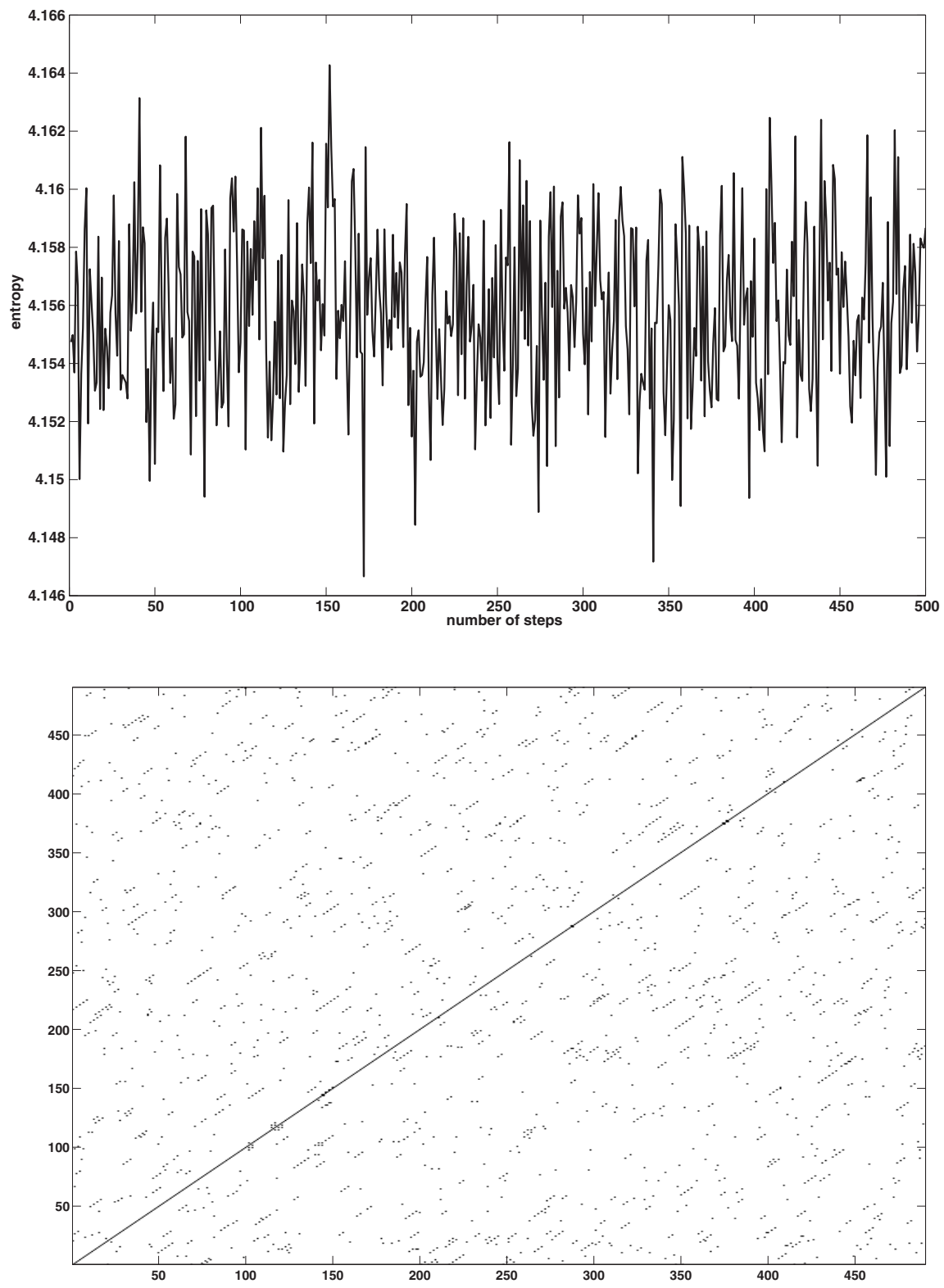

Fig. 14. The same as in Fig.13 but for $p_{a}=0.05$ and $p_{e}=1.00$. 

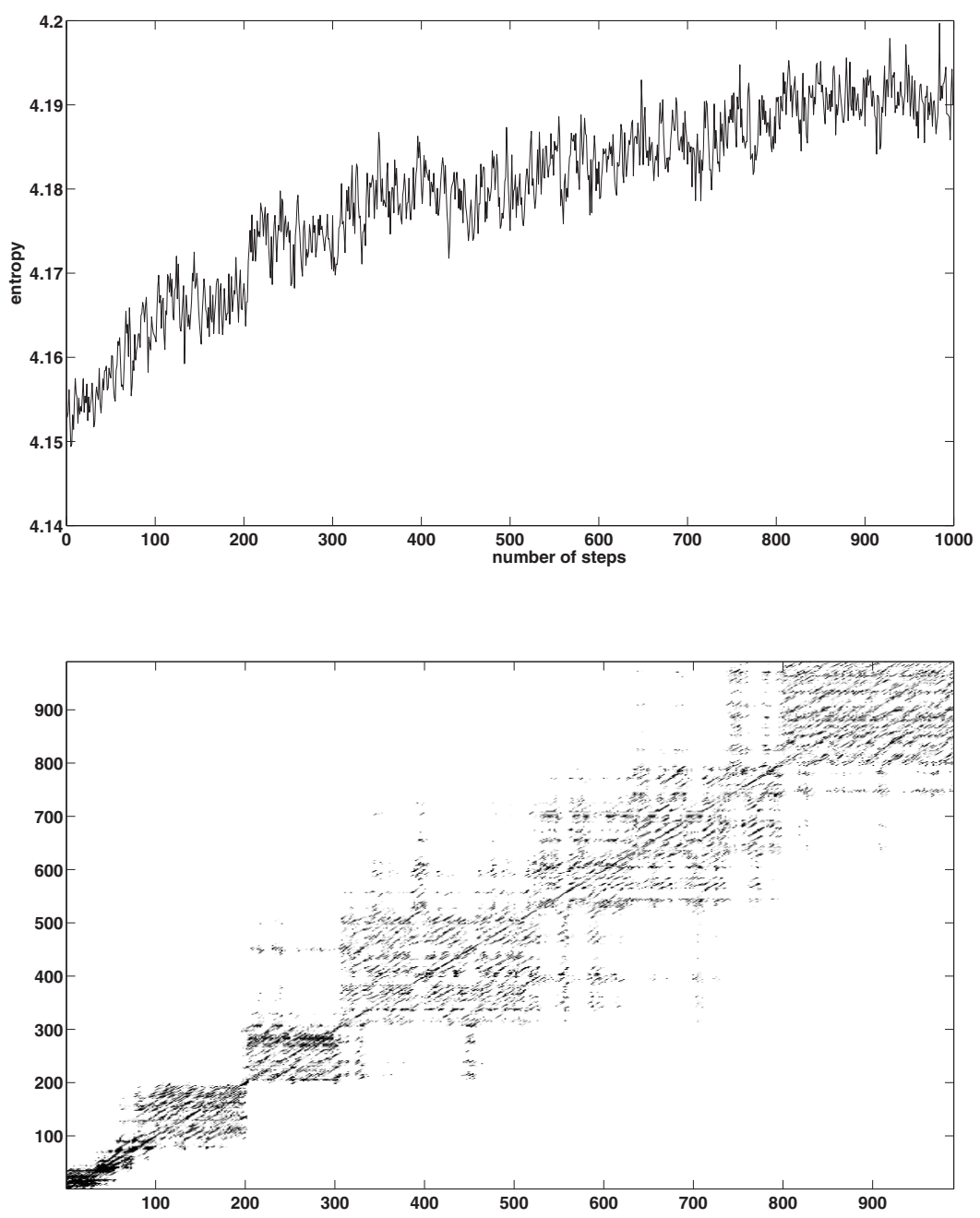

Fig. 15. The same as in Fig.13 but for the cavity with mirrors, $R=0.75, p_{a}=1.00$ and $p_{e}=0.10$.

movement (the excitations "oscillate" from one end of the cavity to another). The leakage of the energy from the system moderates the noise effects and permit uncovering of other features. Moreover, for this case the time-evolution becomes more regular than for the case of constant energy, when many excitations moved randomly inside the cavity.

If energy leakage is increased $(R=0.25)$, the time-evolution of $\mathcal{E}$ looks similar to that discussed for $R=0.75$ (Fig.16 - bottom). However, RP form indicates different character of this evolution. We see that horizontal lines forming rectangular structures again, however 

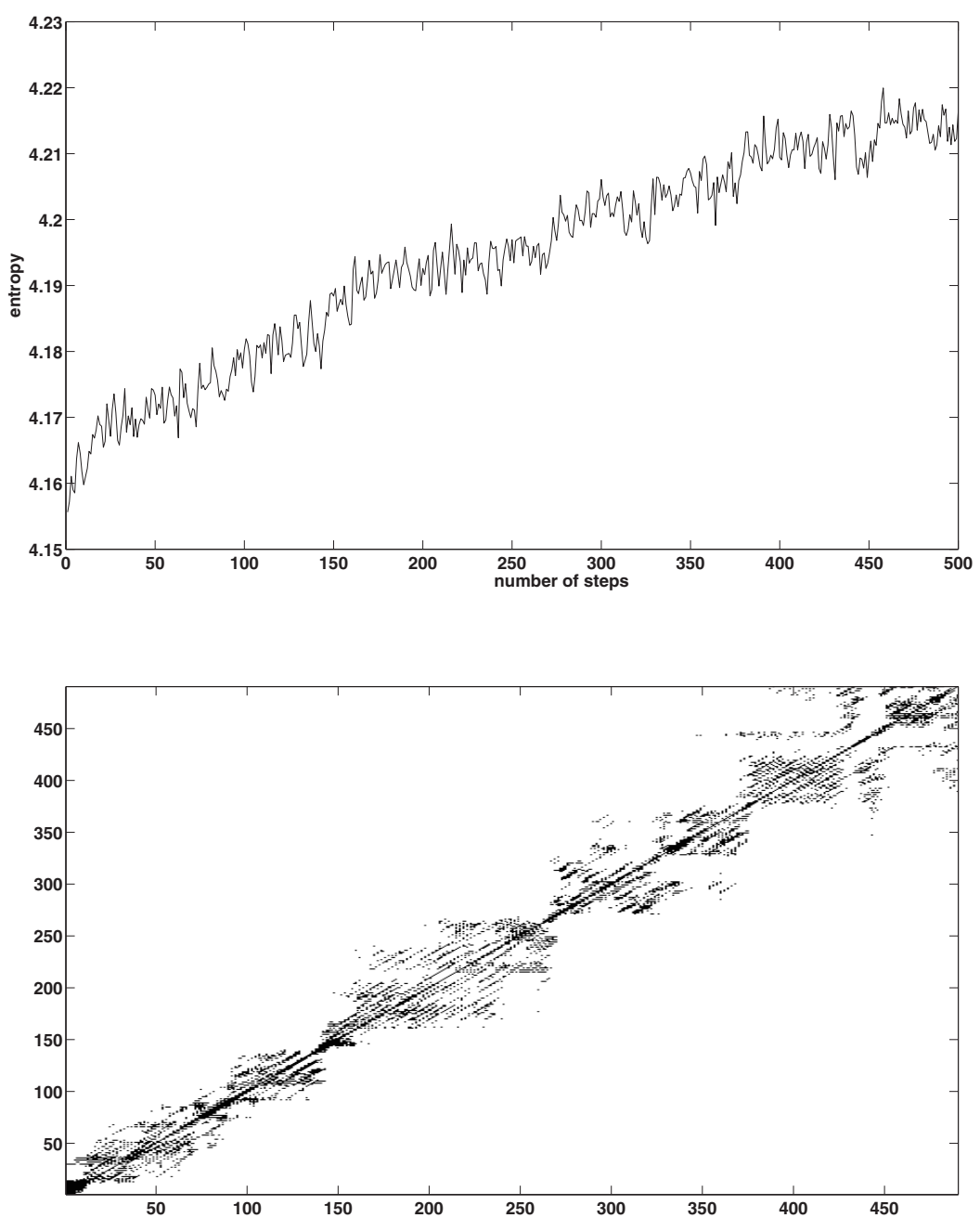

Fig. 16. The same as in Fig.15 but for $R=0.25$.

they are much closer to the main diagonal then in the former case. This means that the system has stabilized itself and there are some regular oscillations inside the cavity. Moreover, there are some diagonal lines, but they are very short and sparse. In fact, during the evolution many energy quanta escapes the system, so fewer excitations remain inside the cavity and their oscillations becomes more distinct.

The results have shown that CA evolution described by RP can exhibit many interesting features. Thanks to the application of RP, we can detect and classify various types of the 
system-evolutions that are invisible and usually remain obscured by a seemingly chaotic behaviour of the system.

\section{Final remarks}

We have shown how the simple rules determining the CA evolution character can lead to a complicated evolution of the model considered. The model discussed here exhibits various features characteristic of the real cavity system. We proposed application of various parameters and investigation methods to characterize the dynamics of the model and hence, its physical properties.

Our model can be treated as a starting point for further investigation. For instance, two or more dimensional systems can be considered instead of one-dimensional one discussed here. Moreover, other mechanisms of energy dissipation than by the "mirrors" considered in this work, and excitation processes also can be taken into regard. However, it should be emphasized that despite the simplicity of the model proposed, it turned out to be a fruitful tool for dynamics investigation.

\section{References}

Allen L. \& Eberly J. H. (2007) Optical Resonance And Two-Level Atoms, Dover Publications, Inc., ISBN 9780486655338, Mineola, New York

Barclay M., Andersen H. \& Simon C. (2010). Emergent Behaviors in a Deterministic Model of the Human Uterus, Reproductive Sciences, Vol. 17, No. 10, 948-954

Bendat J. S. \& Persol A. G. Random Data - Analysis and Measurement Procedures, J. Wiley \& Sons, Inc., ISBN 978-0-470-24877-5, Hoboken, New Jersey

Butler, J. T (1973). A note on cellular automata simulations, Information and Control, Vol. 26, No. 3, 286-295

Cleveland W. S. (1979). Robust locally-weighted re- gression and smoothing scatterplots, J. Am. Stat. Assoc., Vol. 74, No. 368, 829-836

Clevlelend W. S. \& Devlin S. J. (1998) Locally Weighted Regression: An Approach to Regression Analysis by Local Fitting Journal of the American Statistical Association Vol. 83 No. 403, 596-610

Combinido J. S. L. \& Lim M. T. (2010). Modeling U-turn traffic flow, Physica A, Vol. 389, No. $17,3640-3647$

Dabaghian V., Jackson P., Spicer V \& Wuschke K. (2010). A cellular automata model on residential migration in response to neighborhood social dynamics, Math. Comp. Modell. Vol. 52, No. 9-10, 1752-1762

Eckmann J.-P., Kamphorst S. O. \& Ruelle D. (1987). Recurrence Plots of Dynamical Systems, Europhys. Lett. Vol. 4, No. 9, 973-977

Guisado J. L., Jimenez-Moralez F. \& Guerra J. M. (2003). Cellular automaton model for the simulation of laser dynamics, Phys. Rev. E, Vol. 67, No. 6, 066708

Kondrat G. \& Sznajd-Weron K. (2010). Spontaneous Reorientations in a Model of Opinion Dynamics with Anticonformists, Int. J. Mod. Phys. C, Vol. 21, No. 4, 559-566

Kowalewska-Kudłaszyk A. \& Leoński W. (2006). Two-Level Systems, their Evolution and Cellular Automata Method, Acta Phys. Hung. B, Vol. 26, No. 3-4, 247-252

Kowalewska-Kudłaszyk A. \& Leoński W. (2008). Cellular automata and two-level systems dynamics - Spreading of Disorder, J. Comp. Meth. Sci. Eng., Vol.8, No. 1-2, 147-157. 
Ladd A. C. \& Colvin M. E. (1988). Application of lattice-gas cellular automata to the Brownian motion of solids in suspension, Phys. Rev. Lett., Vol. 60, No. 11, 975-978

Lejeune A., Perdang J. \& Richert J. (1999). Application of cellular automata to N-body systems, Phys. Rev. E, Vol. 60 No. 3, 2601-2611

Margolus N., Toffoli T. \& Vichniac G. (1986). Cellular-Automata Supercomputers for Fluid-Dynamics Modeling, Phys. Rev. Lett., Vol. 56, No. 16, 1694-1696

Marwan N., Romano M. C., Thiel M. \& Kurths J. (2007). Recurrence Plots for the Analysis of Complex Systems, Physics Reports, Vol.438, No. 5-6, 237-329.

Kondrat G. \& Sznajd-Weron K. (2009) Spontaneous Reorientations in a Model of Opinion Dynamics with Anticonformists, Int. J. Mod. Phys. C, Vol.21 No. 4, 559-566

Rosin P. L. (2010). Comp. Vision Image Understanding, Vol. 114, No. 7, 790-802

Vichniac G., (1984). Simulating Physics with Cellular Automata. Physica D, Vol.10, No. 1-2, 96-116

Walczak M. \& Leoński W. (2003) A cavity with two-level atoms and cellular automata, Fortchr. Phys., Vol. 51, No. 2-3, 186-189

Zeng H. C., Pukkala T., Peltola H. \& Kellomaki S. (2010) Optimization of irregular-grid cellular automata and application in risk management of wind damage in forest planning, Can. J. Forrest Res., Vol. 40, No. 6, 1064-1075 


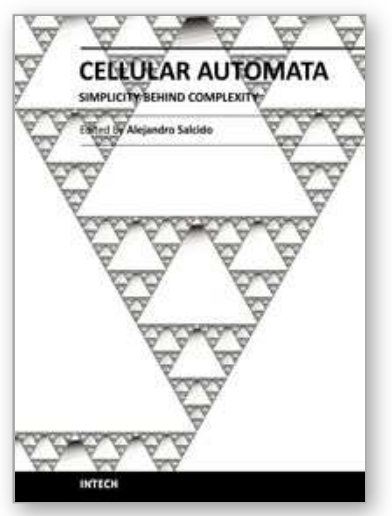

\author{
Cellular Automata - Simplicity Behind Complexity \\ Edited by Dr. Alejandro Salcido
}

ISBN 978-953-307-230-2

Hard cover, 566 pages

Publisher InTech

Published online 11, April, 2011

Published in print edition April, 2011

Cellular automata make up a class of completely discrete dynamical systems, which have became a core subject in the sciences of complexity due to their conceptual simplicity, easiness of implementation for computer simulation, and their ability to exhibit a wide variety of amazingly complex behavior. The feature of simplicity behind complexity of cellular automata has attracted the researchers' attention from a wide range of divergent fields of study of science, which extend from the exact disciplines of mathematical physics up to the social ones, and beyond. Numerous complex systems containing many discrete elements with local interactions have been and are being conveniently modelled as cellular automata. In this book, the versatility of cellular automata as models for a wide diversity of complex systems is underlined through the study of a number of outstanding problems using these innovative techniques for modelling and simulation.

\title{
How to reference
}

In order to correctly reference this scholarly work, feel free to copy and paste the following:

W. Leoński and A. Kowalewska-Kudłaszyk (2011). Cellular Automata - a Tool for Disorder, Noise and Dissipation Investigations, Cellular Automata - Simplicity Behind Complexity, Dr. Alejandro Salcido (Ed.), ISBN: 978-953-307-230-2, InTech, Available from: http://www.intechopen.com/books/cellular-automata-simplicitybehind-complexity/cellular-automata-a-tool-for-disorder-noise-and-dissipation-investigations

\section{INTECH}

open science | open minds

\author{
InTech Europe \\ University Campus STeP Ri \\ Slavka Krautzeka 83/A \\ 51000 Rijeka, Croatia \\ Phone: +385 (51) 770447 \\ Fax: +385 (51) 686166 \\ www.intechopen.com
}

\author{
InTech China \\ Unit 405, Office Block, Hotel Equatorial Shanghai \\ No.65, Yan An Road (West), Shanghai, 200040, China \\ 中国上海市延安西路65号上海国际贵都大饭店办公楼405单元 \\ Phone: +86-21-62489820 \\ Fax: +86-21-62489821
}


(C) 2011 The Author(s). Licensee IntechOpen. This chapter is distributed under the terms of the Creative Commons Attribution-NonCommercialShareAlike-3.0 License, which permits use, distribution and reproduction for non-commercial purposes, provided the original is properly cited and derivative works building on this content are distributed under the same license. 